\title{
IMPROVED SURGICAL OUTCOME OF ENDONASAL DCR WITH PRESERVATION OF NASAL MUCOSAL FLAPS
}

\author{
Anagha Rajguru1, Yogesh Rajguru², Nikhil Vala3 ${ }^{3}$ Ashish Kadam ${ }^{4}$ \\ ${ }_{1}^{1}$ Assistant Professor, Department of ENT, Krishna Institute of Medical College, Karad, Maharashtra. \\ ${ }^{2}$ Consultant Ophthalmologist, Rajyog Hospital, Karad, Maharashtra. \\ ${ }^{3}$ Resident, Department of ENT, Krishna Institute of Medical College, Karad, Maharashtra. \\ ${ }_{4}^{4}$ Resident, Department of ENT, Krishna Institute of Medical College, Karad, Maharashtra.
}

\section{BACKGROUND}

ABSTRACT

Dacryocystorhinostomy (DCR) has distinct advantage over external DCR. It is a highly evolving endoscopic procedure of management of dacryocystitis. It is a surgery in which a fistulous tract is created between the lacrimal sac and nasal cavity when epiphora does not respond to medications and repeated syringing of nasolacrimal duct. The advantages of endonasal DCR over external DCR are- there is no ugly scar on the face, less intraoperative bleeding, day care procedure, simple and less time consuming procedure and safe but skilful surgery. The long-term success rate of external DCR in dedicated oculoplastic census is very high $(90 \%-95 \%)$. But although the better success rate is seen, which is close to $100 \%$ with some modifications in endonasal DCR techniques. Even if there is recurrence of epiphora, it is again correctable fully with no residual effects.

Aim and Objective- To compare outcome of new method of endonasal DCR.

\section{MATERIALS AND METHODS}

A non-randomised controlled trial was conducted from February 2014 to March 2017. A sample size of 360 was taken for convenience in 360 patients, of which 108 (30\%) were males and 252 (70\%) were females. We performed few cases with removal of nasal mucosal flap and rest of the cases with preservation of nasal mucosal flaps.

\section{RESULTS}

360 patients were operated for chronic dacryocystitis. 200 patients were operated with modified technique of preserving nasal mucosal flap and repositioning it at the end of surgery with the intention of no bare bone should be left behind named as Group A. 160 patients were operated with routine method where nasal mucosa was removed named as Group B. No intraoperative complications were encountered. Average follow-up duration was 24 months. During this period, the elimination of subjective symptoms and patency of the nasolacrimal passage confirmed by saline irrigations and endoscopic visualisation of ostium were accepted as surgical success. Surgical success was achieved in 197 (98.5\%) out of the 200 patients in group A. In group B, success was achieved in 147 (91.8\%) out of 160. Our study shows increase in success rate when technique is modified with repositioning of nasal mucosal flap on bare bone, which reduces chances of synechiae and granulation postoperatively.

\section{CONCLUSION}

Comparative study of these two methods showed that there is marked improvement in surgical outcome of endonasal DCR when the nasal mucosal flap is preserved.

\section{KEYWORDS}

Endoscopic Endonasal Dacryocystorhinostomy, Nasal Mucosal Flap Preserve, Granulation Tissue, Surgical Access.

HOW TO CITE THIS ARTICLE: Rajguru A, Rajguru Y, Vala N, et al. Improved surgical outcome of endonasal DCR with preservation of nasal mucosal flaps. J. Evolution Med. Dent. Sci. 2018;7(14):1765-1768, DOI: 10.14260/jemds/2018/398

\section{BACKGROUND}

Endonasal DCR is now-a-days a widely accepted procedure of chronic dacryocystitis, in which a tract is created between the lacrimal sac and nasal cavity to drain lacrimal fluid which is collected in the sac because of NLD obstruction.(1-3) This procedure can be carried out in two ways, either by removing nasal mucosa or by preserving it and replacing it back on the bare bone at the end of the procedure.

'Financial or Other Competing Interest': None.

Submission 19-02-2018, Peer Review 12-03-2018,

Acceptance 18-03-2018, Published 02-04-2018.

Corresponding Author:

Dr. Anagha Rajguru,

Rajyog Hospital,

Near Popatbhai Petrol Pump,

Shaniwar Peth, Karad-415110,

Dist. Satara, Maharashtra.

E-mail: anagha.ent134@gmail.com

DOI: $10.14260 /$ jemds $/ 2018 / 398$
Actually, endonasal dacryocystorhinostomy is not a new concept. First it was described by Caldwell Luc in 1893 and later in 1911 by West; however, the procedure did not gain popularity because of difficult visualisation of endonasal structures during surgery. With the upgradation of technology, the visualisation of nasal structures during surgery improved results markedly.

Three groups of procedures are currently practiced, external DCR, endoscopic DCR with contact laser surgical endoscopic DCR without lasers. External DCR has remained the most successful surgical procedure of nasolacrimal duct obstruction with success rate of 90\% - 95\%.(4) Important precision for achieving this success rate are exposure of entire lacrimal sac by wide bone removal.(5) Anastomosing the lacrimal sac mucosa and nasal mucosa, avoidance of keeping bare bone left behind.

\section{Objective}

To compare outcome of new method of endonasal DCR. 


\section{MATERIALS AND METHODS}

A non-randomised controlled trial was conducted from February 2014 to March 2017. A sample size of 360 was taken for convenience. In 360 patients, of which 108 (30\%) were males and 252 (70\%) were females.

Patients aged varied from 05 - 80 years, those subjects who underwent primary or revision intranasal endoscopic endonasal DCR surgery. These patients were usually referred by ophthalmologists. With history of epiphora and nasolacrimal duct obstruction. The subjects were assigned to either group A or group B depending on preoperative nasal mucosal flap position. Group A patients were treated with repositioning of nasal mucosal flap on bare bone and group $B$ patients were treated with the removal of nasal mucosal flap and the bare bone was covered with Abgel.

In our series there were 10 cases of acute dacryocystitis, for which surgery was planned after 1 week of antibiotic treatment. 6 cases of lacrimal fistula, 2 cases of lacrimal abscess were also treated with endonasal DCR. All patients underwent complete preoperative ophthalmological and endoscopic nasal examination including lacrimal syringing, regurgitation testing and endoscopic nasal examination helped to diagnose septal deviation where patients were subjected to septal deviation correction for better access to operative site. All cases were operated under local anaesthesia with sedation.

The nose was prepared using cotton strips soaked in $4 \%$ xylocaine and adrenaline in ratio of 4: 120 minutes prior to surgery. Adequate decongestion and mucosal anaesthesia helps easy access and bloodless field. 2\% xylocaine with 1:200000 adrenaline was injected submucosally into the lateral nasal wall superior and anterior to the attachment of the middle turbinate and then along with maxillary line.

\section{Surgical Technique}

An incision was made in lateral wall of nose with the help of sickle knife. Inferiorly based mucosal flap was created by incision that began approximately $5 \mathrm{~mm}$ above the insertion of middle turbinate on the lateral nasal wall and was extended horizontally $8 \mathrm{~mm}$ anterior to the middle turbinate. A vertical incision was made from anterior end of the superior horizontal incision with keeping mucosal attachment with inferior turbinate intact. Same parallel incision was made posteriorly that extends from axilla of middle turbinate above inferior turbinate below. A Freer's periosteal elevator was used to strip the mucosa with underlying mucoperiosteum from the underlying bone, flap everted on the inferior turbinate. This flap was preserved in group A patients. In group B patients, the mucosal flap was peeled off by continuing anterior incision posteriorly to the insertion of uncinate process above the inferior turbinate. Except mucosal flap preservation, rest of the operative procedure was the same of both the groups. The thin lacrimal bone and thick maxillary bone was identified.

An osteotomy was performed with straight $2 \mathrm{~mm}$ SmithKerison punch forceps. An angled punch was used to remove bone at the upper limit of the sac. Sometimes removal of thick bone from frontal process of maxilla was required for which a drill with 1 - $2 \mathrm{~mm}$ cutting burr was used. The exposure of the inferior and superior parts of the lacrimal required a large osteotomy (at least $15 \mathrm{~mm} \times 10 \mathrm{~mm}$ ). Intraoperative removal of the bone surrounding the common canaliculus at the superior third of the lacrimal sac prevents the bone to act as a scaffold for fibroblast growth, ultimately leading to obliteration of the scar. A metallic lacrimal probe was passed medially through the inferior lacrimal canaliculi and was gently passed to tent the sac. An incision was made in sac was with 12 number blade and injury to the sac lumen was avoided. The vertical incision was extended superiorly and inferiorly. The lacrimal canal was irrigated with saline solution. In group A the nasal mucosal flap was replaced back on bare bone, taking care not to cover sac area. The surgical site was packed with a small piece of merocele to hold the flap in position. In group B, the nasal mucosal flap was trimmed with scissors. Thus, the postoperative complication of formation of scar and granulation tissue in bare bone area was minimised.

\section{Postoperative Care and Follow-Up}

Patients were discharged uneventfully the day after surgery with oral antibiotics and analgesics for 1 week with antibiotic and steroid eye drops for 1 month. The merocele was usually removed at the time of discharge. Patients were advised to avoid nose blowing for 7 weeks as to avoid nasal haemorrhage and orbital emphysema. Patients were followed up weekly for a month.

\section{Statistical Analysis}

Data of both groups were compared and analysed for statistical significance with Chi-square test using IBM SPSS Statistics Version 20 software.

\section{RESULTS}

We analysed the results by company of the surgical outcomes in the two groups. Data regarding the patients' age, sex, side of operation, type of surgery (with or without mucosal flap) and post-surgical complications were recorded.

In the series of 360 patients, 108 patients were male (30\%) and 252 were female patients (70\%).

The youngest patient found in the study was 5 -year-old boy and the eldest was 86-year-old female. Age ranged from 5 to 90 years.

Of these 360 procedures, 324 were primary (89.72\%) and 36 were revision DCR (10\%). All patients complained of preoperative epiphora. All selected cases were of unilateral chronic dacryocystitis.

In group A, septoplasty was required in 6 cases. In group $B$, septoplasty was performed in 2 patients. The patient who had other nasal pathologies were not included in the study.

The patients were followed up to 6 to 24 months after their operation. Overall, the success rate of the procedure in group A (98.5\%) was significantly higher than success rate of group B $(91.8 \%)$ in the terms of subjective improvement in the eye watering and swelling at the medial canthus of eye. (Chi-square $=9.186, p=0.0024)$. Patients were also evaluated endoscopically to visualise the patent stoma and also with syringing.

\begin{tabular}{|c|c|c|}
\hline Sex & No. of Patients & Percentage \\
\hline Male & 108 & $30 \%$ \\
\hline Female & 252 & $70 \%$ \\
\hline Total & $\mathbf{3 6 0}$ & $\mathbf{1 0 0 \%}$ \\
\hline \multicolumn{3}{|c|}{ Table 1 } \\
\hline
\end{tabular}




\begin{tabular}{|c|c|c|c|}
\hline Group & $\begin{array}{c}\text { Total No. } \\
\text { of Cases }\end{array}$ & $\begin{array}{c}\text { Successful } \\
\text { Cases }\end{array}$ & Percentage \\
\hline A & 200 & 197 & $98.5 \%$ \\
\hline B & 160 & 147 & $91.8 \%$ \\
\hline \multicolumn{4}{|c|}{ Table 2 } \\
\hline
\end{tabular}

\section{DISCUSSION}

Epiphora is an annoying symptom, which is embarrassing to the patient both socially and functionally. Epiphora resulting from obstruction of nasolacrimal duct has two widely accepted treatment modalities, external and endonasal DCR. Endonasal DCR has well known advantages over the standard external DCR, like it avoids facial scaring. Division of the medial canthal ligament and disruption of the pump action of the lacrimal sac. It has minimal morbidity and less risk of intraoperative bleeding. It also enables direct access to the rhinostoma site, reducing tissue injury.(6) It has shorter operating time and easy access route.(7) The main advantage of external DCR is visualisation of the anatomy allowing precise of the bone in the lacrimal fossa and exact anastomosis of the nasal mucosa and lacrimal sac wall.

Endoscopic surgeon should have a good knowledge of the anatomy of lacrimal sac and duct within the nose, in order to obtain optimum results comparable to those of external DCR. The disadvantage of endoscopic endonasal DCR are the difficulty in detecting pathological conditions of the nasolacrimal sac or duct, difficulty or impossible suturing of the adjacent flaps of the lacrimal sacs and nasal mucosa, the need for meticulous haemostasis (which is essential of endoscopic visibility) and a lower success rate than that of external DCR. $(8,9)$

Durrasula and Gatland reported the formation of granulation tissue is caused by bare bone.(10)

$\mathrm{T}$ Siroasetal and Onerei and Colleagues describe an approach that involves the preservation of nasal mucosa and cutting of anterior and posterior flaps in the lacrimal sac to achieve mucosal and lacrimal sac anastomosis as the conclusion of the procedure.(11,12) Those authors reported a success rate of $90 \%$ when that technique was used.

Tsirbas and Wormald(13) stated that the key to successful endoscopic DCR is to fully expose the lacrimal sac and marsupialised into that lateral nasal wall with the nasal and lacrimal mucosa in apposition allowing healing by primary intention rather than formation of granulation tissue, reducing the risk of closure of the sac opening into the nose.

Our technique involves creation of large bony ostium and a large inferior flap, which was used to cover bare lacrimal bone at the end of surgery.

Mahendran and colleagues explained that the mucosa and bone over lacrimal duct and sac must be removed to access the lacrimal sac. Therefore, a portion of the lacrimal bone remains exposed at the completion of the operation. This can lead to the formation of granulation and scar tissue around the lacrimal window. To prevent the complication Mahendran and colleagues have used a free mucosal flap to cover the bare bone in patients underlying endoscopic endonasal DCR.(14) We support the combination of large bony ostium, which is a proposal of Wormald and covering bare lacrimal bone with preserved nasal mucosa at the end of surgery as explained by Mahendran and Colleagues help to achieve post-op success rate almost near $99 \%$.

\section{CONCLUSION}

We have modified the nasal mucosal incision on the lateral nasal wall, performing inferiorly based mucosal flap keeping flap, all attachments intact with that of inferior turbinate, which was preserved and replaced back on bare lacrimal bone at the end of surgery. This technique promotes a quick and controlled epithelialisation of the lateral nasal wall, allowing controlled healing of surgical site. The success rate of this technique is $98.92 \%$ and is better than external DCR.

We conclude that low complication and high success rate indicate that endonasal DCR with a large bony ostium and large nasal mucosal flap covering bare lacrimal bone can be considered as superior technique in treatment of lacrimal system obstructions. We strongly believe that our modified technique emulates the external approach through a bare minimal and less invasive approach, but with better outcomes.

\section{REFERENCES}

[1] Unlu HH, Toprak B, Aslan A, et al. Comparison of surgical outcomes in primary endoscopic dacrocystorhinostomy with and without silicone intubation. Ann Otol Rhinol Laryngol 2002;111(8):704-9.

[2] Kupper DS, Demarco RC, Resende R, et al. Endoscopic nasal dacrocystorhinostomy: results and advantages over the external approach. Braz J Otorhinolaryngol (Engl Ed) 2005;71(3):356-60.

[3] Wormald PJ. Powered endoscopic dacrocystorhinostomy. Laryngoscope 2002;112(1):69-72.

[4] Hartikainen J, Antila J, Varpula M, et al. Prospective randomized comparison of endonasal endoscopic dacrocystorhinostomy and external dacrocystorhinostomy. Laryngoscope 1998;108(12):1861-6.

[5] Rajguru AY, Rajguru YR. Use of mytomicin in enonasal DCR. JEMDS 2014;3(20):5642-4.

[6] Eloy PH, Bertrand B, Martinez M, et al. Endonasal dacrocystorhinostomy: indications, technique and results. Rhinology 1995;33(4):229-33.

[7] Whittet HB, Shun-Shin GA, Awdry P. Functional endoscopic transnasal dacrocystorhinostomy. Eye (Lond) 1993;7(Pt 4):545-9.

[8] Zilelioglu, Tekeli O, Ugurba $\mathrm{SH}$, et al. Results of endoscopic endonasal non-laser dacrocystorhinostomy. Doc Opthalmol 2002;105(1):57-62.

[9] Mandeville JT, Woog JJ. Obstruction of lacrimal drainage system. Curr Opin Opthalmol 2002;13(5):303-9.

[10] Durrasula VS, Gatland DS. Endoscopic transnasal dacrocystorhinostomy. J Laryngolotol 1989;103:5857.

[11] Tsirbas A, Wormald PJ. Endonasal dacrocystorhinostomy with mucosal flap. Am J Ophthalmol 2003;135(1):76-83.

[12] Onerci M, Orhan M, Ogretmenoglu O, et al. Long-term results and reasons for failure of intranasal endoscopic dacrocystorhinostomy. Acta Otolaryngol 2000;120(2):319-22. 
[13] Tsirbas A, Wormald PJ. Mechanical endonasal dacrocystorhinostomy with mucosal flap. $\mathrm{Br}$ J Opthalmol 2003;87(1):43-7.
[14] Mahendran S, Stevens-King A, Yung MW. How we do it: the viability of free mucosal grafts on exposed bone in lacrimal surgery - a prospective study. Elin Otolaryngol 2006;31(4):324-7. 